wards due to resource constraints. We evaluated a mobile phone text message intervention (YourCall) designed to overcome this challenge.

Methods Of 1564 potentially eligible injured patients aged 1669 years recruited from the three trauma admitting public hospitals in Auckland and screened using the Alcohol Use Disorders Identification Test (AUDIT), 598 were identified as moderate risk drinkers and enrolled in a parallel, single-blind RCT. The intervention group received 16 text messages incorporating BI principles in the four weeks after hospital discharge. Controls received one text message acknowledging participation in the study. Primary outcomes comprised differences in hazardous alcohol use (AUDIT-C) between the intervention and control groups at 3, 6 and 12 months following the injury. Data were analysed using a mixed-effects model for repeated measures.

Results Baseline features were similar in both groups (71\% males; mean age 34 years; 21\% Māori, 62\% European; mean AUDIT-C 6.8). Significant reductions in hazardous alcohol use during follow-up were observed in both groups. A small but significantly lower risk of hazardous drinking was evident in the intervention compared with control group (least square means difference: -0.322 ; 95\% CI: $-0.636,-0.008)$. This effect was maintained across the 12-months of follow-up and similar among Māori and non-Māori (interaction $\mathrm{p}=0.257$ ).

Conclusions The effect of the Your Call intervention was similar to most standard BIs despite its modest intensity and the restriction of this trial to moderate risk participants only. Text interventions are scalable low cost approaches that can overcome barriers and inequities in implementing BIs in trauma care settings. The expanding cell phone coverage makes this modality particularly salient in economically disadvantaged groups and low- and middle-income countries.

\section{CHALLENGES IN THE IMPLEMENTATION OF CONCUSSION GUIDELINES IN COMMUNITY SPORT}

${ }^{1}$ Caroline F Finch, ' Joanne Kemp, ${ }^{1}$ Peta White, ${ }^{2}$ Joshua Newton. ${ }^{1}$ Federation University Australia, Australia; ${ }^{2}$ Deakin University, Australia

\subsection{6/injuryprev-2016-042156.260}

Background Concussion in sport has become a major injury prevention priority worldwide because of its potential severity. International consensus guidelines for concussion in sport have been developed and promoted. To date, there has been little consideration of the barriers that might influence how well these guidelines are adopted and implemented in practice, especially in community sport.

Methods A self-report online survey was developed from Theory of Planned Behaviour constructs. 111 coaches and sports trainers from community Australian Football and Rugby League completed both pre- and post-season surveys and data on their attitudes towards using concussion guidelines was extracted. Postseason attitudes were assessed relative to pre-season attitudes and whether or not responders had used the guidelines during the intervening playing season. Specific feedback about any challenges in using the guidelines was also sought.

Results $71 \%$ of respondents had used the guidelines. Post-season attitude was related to pre-season attitude $(p=0.002)$, football code $(\mathrm{p}=0.015)$, and team role $(\mathrm{p}=0.045)$. There was a significant interaction between team role and guideline use ( $p=0.012$ ), with coaches who had used the guidelines, and sports trainers who had not, reporting more positive post-season attitudes. Implementation challenges included disputing of decisions about return-to-play by players, parents, and coaches, and a perceived lack of time. Recommendations for improved guideline materials included using larger fonts and formal witnessing of advice given.

Conclusions This is the first study to examine the implementation of concussion guidelines in community sport. Information from those who attempt to use them is valuable to inform the refinement of implementation and dissemination processes around concussion guidelines across sports. New education should be developed about the importance of advice given to parents/players them by those who follow these guidelines.

\section{THE DISTRICT OF COLUMBIA CONCUSSION CARE \& TRAINING PROJECT: A MODEL FOR YOUTH CONCUSSION EDUCATION}

${ }^{1}$ Andrew E Lincoln, ${ }^{1}$ Justin B Cooper, ${ }^{1}$ Ethan A Urbansky, ${ }^{2}$ Gerald A Gioia, ${ }^{2}$ Shireen Atabaki, ${ }^{2}$ Catherine McGill, ${ }^{2}$ Maegan Sady, ${ }^{1}$ Michael Yochelson, ${ }^{1}$ Elizabeth Delasobera, ${ }^{1}$ Jessica Clark. ${ }^{1}$ MedStar Sports Medicine, Washington, $D C$; ${ }^{2}$ Children's National Medical Centre, Washington, DC

\subsection{6/injuryprev-2016-042156.261}

Background The District of Columbia's "Concussion Protection Act of 2011" legislation requires training of youth sports program stakeholders to ensure concussion awareness and training to properly recognise, treat, and recover from a concussion. A partnership between the Children's National Medical Centre, MedStar Sports Medicine and community stakeholders was established in March 2015 to develop an infrastructure and pathways for appropriate training and documentation of compliance.

Methods The Concussion Care and Training Program (CCTP) included four priority areas: Creating Awareness, Concussion Training, Policy and Protocol Development, and Evaluation and Performance Measurement. The CCTP team implemented a combined clinical and public health approach to provide comprehensive, up-to-date training and documentation. Partnerships were established with program administrators in schools, recreation programs, and community associations to establish training needs and maximal accessibility of target audiences. Specific products were developed and disseminated across the community.

Results The CCTP performed 29 training activities to over 6000 participants. Of the 225 training participants who completed the program assessments, the largest affiliations were associated with Children's School Services (24\%), Public Schools (17\%), and Private Schools (14\%). The most common participant roles were School Nurses (44\%) and Coaches (33\%). Overall, 40\% of training participants were aware of the DC concussion law prior to the training. Only $41 \%$ of coaches and $32 \%$ of parents claimed to be aware of the law.

Conclusions This model program reached stakeholders of all ages and levels of youth athletes across diverse communities to provide concussion education/training, media outreach and public awareness. This model may be used by other localities to increase youth sports concussion awareness and training among community participants. 